ARTICULO DE INVESTIGACION

\title{
Evaluación del manejo forestal regular e irregular en bosques de la Sierra Madre Occidental
}

\section{Regular and irregular forest management evaluation of the Sierra Madre Occidental forests}

\author{
José Ciro Hernández-Díaz1 ${ }^{*}$, José Javier Corral-Rivas², Andrés \\ Quiñones-Chávez ${ }^{3}$, Jeffrey R. Bacon-Sobbe 1 \\ y Benedicto Vargas-Larreta 4
}

\begin{abstract}
RESUMEN
En este trabajo se evaluó la recuperación del volumen de Pinus en pie después de una corta, en bosques tratados con los sistemas de manejo regular e irregular. Para ello se revisaron los antecedentes de cinco predios manejados con el Método Mexicano de Ordenación de Bosques Irregulares (MMOBI) y doce predios manejados con el Método de Desarrollo Silvícola (MDS). Además, en estos predios, en el año 2004 se muestrearon 12 unidades básicas de manejo del MMOBI y 34 del MDS. La intensidad de muestra fue del $5 \%$ y se usaron sitios circulares de $1000 \mathrm{~m}^{2}$. De los 17 predios estudiados, en 11 ya se había cambiado de método de planeación para el año 2004, en ocho se observó que hubo cambios en la delimitación de las unidades básicas de manejo en los programas posteriores al de la anualidad 1995-1996. Se encontró que en el año 2004, aunque ya había transcurrido casi un ciclo de corta, el volumen por hectárea en seis de las 12 unidades básicas de manejo analizadas del MMOBI y en 19 de las 34 unidades de manejo del MDS fue significativamente diferente $(\alpha=5 \%)$. Estas diferencias se atribuyen a los cambios en la delimitación de las unidades básicas de manejo y a una baja precisión de los inventarios, causada por: i) inadecuada intensidad de muestreo, ii) errores en la estimación de las categorías diamétricas y de altura y iii) uso de factores de área basal inadecuados.

PALABRAS CLAVE:

Factor de área basal, manejo de bosques regulares e irregulares, Método de Desarrollo Silvícola, Método Mexicano de Ordenación de Bosques Irregulares, rodalización.
\end{abstract}

\begin{abstract}
The recovery of standing Pinus stock after a harvest in forests treated under irregular and regular forest management systems was evaluated. The management history of five forest properties managed under the Mexican Management Method of Irregular Forests (MMOBI) and twelve under the Silvicultural Development Method (MDS) during the period 1995-96 was analyzed. In addition, in 2004 within these forest properties, 12 basic MMOBI forest management units and 34 basic MDS units were tera a Mazatlán; CP 34120, Durango, Dgo., México.

2 Facultad de Ciencias Forestales. Universidad Juárez del Estado de Durango. Río Papaloapan y Blvd. Durango; Col. Valle del Sur; CP 34120; Durango, Dgo., México.

3 Campo experimental Valle del Guadiana, INIFAP; Km 5.5, Carretera a El Mezquital; Durango, Dgo.

4 Instituto Tecnológico Forestal núm. 1. Mesa del Tecnológico s/n. AP 2, CP 34950, El Salto, P.N., Durango, México.

* $\quad$ Autor para correspondencia: Tel y Fax: +52-618-8271215. Correo electrónico: jciroh@ujed.mx
\end{abstract}


sampled with an intensity of $5 \%$ of their total area, using $1000 \mathrm{~m}^{2}$ circular plots. Of the 17 properties managed, either with MMOBI or MDS in 1995-96, 11 had shifted to another forest planning method by the year 2004. In eight of the 17 properties, management programs for the harvest cycle following the one containing the data for the period 199596 indicated that there were modifications to the delimitation of the basic forest management units. It was found out that by 2004 , even though a cutting cycle had almost culminated, the volume per hectare in six of the $12 \mathrm{MMOBI}$ units analyzed and in 19 of the 34 MDS units analyzed were significantly different $(\alpha=$ $5 \%$ ). The differences are attributed to the changes in delimitation of the basic forest management units and to a low precision of the forest inventories, due to: a) inadequate sampling intensity, b) errors in the estimation of diameters and heights classes, and c) the use of inadequate basal area factors.

\section{KEYWORDS:}

Basal area factor, stands delimitation, regular and irregular forest management, MMOBI, MDS.

\section{INTRODUCCIÓN}

Según Mendoza y Rodríguez (1959), hasta el año de 1940 la posibilidad de corta de madera en los bosques de México se calculaba con diversas fórmulas de origen europeo, como la de Heyer, el método Francés y el de Melard. En 1941, en la primera Convención Nacional Forestal, Carlos Treviño Saldaña, basado en intensos estudios biométricos realizados en Durango y Puebla, manifestó que estos métodos eran imprecisos al aplicarse a los bosques mexicanos, que eran casi vírgenes. En este evento, Treviño Saldaña propuso su fórmula para calcular la posibilidad de corta, basada en el incremento de la masa, magnitud de las existencias reales actuales y las normales, y en el periodo de planeación propuesto por el silvicultor para que el monte alcanzara la normalidad. La innovación principal fue que dichas variables debían deducirse de las condiciones reales de cada bosque a ser manejado (Mendoza y Rodríguez, 1959).

En 1944, el Servicio Forestal Oficial de México, tratando de asegurar que el aprovechamiento de cualquier bosque en el país fuera persistente, remarcó la importancia de calcular su incremento anual y con base en éste, en la fórmula del interés compuesto y en una intensidad de corta no mayor al $35 \%$ de las existencias reales totales (ERT), poder calcular un ciclo de corta que permitiera por lo menos la recuperación de los volúmenes cortados en cada rodal intervenido. Sin embargo, estos lineamientos oficiales no especificaban un plan de desarrollo del bosque ni lo que se esperaba lograr con su manejo en el mediano y largo plazo (Mendoza y Rodríguez, 1959). Esta deficiencia se superó en 1951, cuando la Dirección Técnica de la Unidad Industrial de Explotación Forestal de Atenquique, Jalisco, presentó su proyecto general de ordenación de bosques. En este documento se realizó un análisis de los lineamentos oficiales de manejo, con lo cual surgió el Método Mexicano de Ordenación de Montes (MMOM), que en los siguientes 25 años se generalizó para casi todos los bosques del país. Una característica relevante del MMOM, no prevista en la disposición oficial citada, consistió en hacer variar la intensidad de corta en cada predio o rodal según su porcentaje de incremento corriente, dado un ciclo de corta general previamente determinado (Mendoza y Rodríguez 1959 y Torres, 1999).

A principios de los años de 1980 , el nombre del MMOM cambió a MMOBI (Método Mexicano de Ordenación de Bosques Irregulares), al incorporarse el criterio de utilizar como referencia la Curva de Liocourt (SEMARNAP y AMPF, 1998) que relaciona el número de árboles de diferentes categorías diamétricas que 
debe haber en un bosque normal por unidad de superficie, para guiar la estructura de las masas manejadas hacia la meta ideal de bosque irregular normal (Torres, 1999). Tanto en el MMOBI como en su antecesor, todos los rodales se manejan bajo el sistema de bosques irregulares, aprovechándolos mediante el tratamiento único de "cortas de selección".

En los años de 1970, se observó que algunos bosques nuevos se originaron a consecuencia de las cortas intensivas efectuadas a principios del siglo XX. A estos bosques se les llamaba "de segundo crecimiento" y semejaban estructuras regulares o tendientes a la regularidad, por lo que algunos técnicos impulsaron lo que en aquel tiempo se denominó "silvicultura intensiva", conocido después como el Método de Desarrollo Silvícola (MDS). En este sistema de manejo se aplican varias "cortas de aclareo" en la etapa de crecimiento rápido del bosque, y al final del turno se aplica una corta intensiva llamada "corta de regeneración" (dejando en pie solamente árboles padres), para promover que se establezca la regeneración natural, misma que es liberada en el siguiente ciclo de corta. El objetivo final de esta secuencia de tratamientos es llevar a los bosques desde su estructura original (un tanto irregular) hacia una estructura regular, a través de la ejecución de un turno (Cano, 1988).

En la década de 1980 el MDS casi llegó a generalizarse en todo el estado de Durango, remplazando en muchos casos al MMOBI. Sin embargo, en esa misma década algunos técnicos formularon la hipótesis de que no era correcto generalizar el manejo irregular, ni tampoco el manejo regular a todos los bosques, pues las condiciones que se presentan en cada predio y en cada rodal son diversas. Empezaron entonces a buscarse métodos mixtos que permitieran aplicar en un mismo predio y en cada rodal los conceptos de manejo regular o irregular, dependiendo de su topografía, clima y vegetación, así como de los objetivos buscados.

Es así como en Durango se aplican ahora, además del MMOBI y el MDS, cuatro métodos mixtos, que combinan en el mismo predio los sistemas de manejo de bosque regular e irregular, que son: el Sistema de Conservación y Desarrollo Silvícola (SICODESI), el Sistema Integral de Manejo de Bosques de la Unidad Santiago (SIMBUS), el Sistema de Manejo Integral Forestal de Tepehuanes (SMIFT) y el llamado Método Mixto.

Esta variedad de métodos indica que el manejo forestal en Durango se ha ido refinando y es más específico en las últimas décadas. Sin embargo, al igual que en otras partes del mundo, en Durango poco se ha estudiado en relación a la respuesta del bosque a cada método o sistema de planeación, para ayudar a definir cuáles prácticas silvícolas han sido apropiadas y de qué manera influyen para lograr la sustentabilidad en el manejo forestal (Pélissier et al., 1998; Gadow et al., 2004; Corral et al., 2005 y Solís et al., 2006). Como una contribución a lo anterior, el presente trabajo tiene como objetivo evaluar técnicamente el uso de los sistemas de manejo regular e irregular que fueron aplicados en bosques del estado de Durango desde 1990 hasta el año 2004. En particular, el enfoque del estudio fue evaluar el grado de recuperación del volumen en pie del género Pinus, a nivel de unidad de manejo tratada con el MMOBI y con el MDS. 


\section{METODOLOGÍA}

\section{Ubicación}

La Sierra Madre Occidental del estado de Durango, México, se localiza entre las coordenadas geográficas $26^{\circ}$ $50^{\prime}$ y $22^{\circ} 17^{\prime}$ de latitud norte y $107^{\circ} 09^{\prime}$ y $102^{\circ} 30^{\prime}$ de longitud oeste (SARH, 1994). Esta región se caracteriza por presentar climas templados fríos, con bosques de diversos tipos.

El estudio se realizó en una muestra de predios bajo aprovechamiento forestal comercial, constituidos principalmente por bosques mixtos de pino-encino (Pinus-Quercus) o de encino-pino (Quercus-Pinus); con menor abundancia se aprovechan bosques de coníferas donde predomina el género Pinus y también algunos bosques de hojosas, en especial Quercus (González et al., 2007). En la figura 1 se ilustra la ubicación aproximada de los predios que constituyeron la muestra, aclarando que para realizar este trabajo se analizaron únicamente los casos que en la anualidad 1995-1996 se manejaban con los métodos MMOBI y MDS.

\section{Selección de los predios y sistemas de manejo a estudiar}

Se tomó como base de análisis la anualidad 1995-1996, asumiendo que el tipo de actividades de manejo que se realizaron en esa anualidad fueron similares a las que se efectuaron en cualquier otra anualidad del ciclo de corta vigente en los años de 1990. En los archivos de la delegación federal de la Secretaría del Medio Ambiente y Recursos Naturales (SEMARNAT), se encontró que entre los meses de julio de 1995 y junio de 1996 se expidieron autorizaciones a 229 predios para realizar algún tipo de aprovechamiento persistente.

En un estudio más amplio, del cual se desprende el presente reporte, se analizaron 31 predios, distribuidos de tal manera que representaron por lo menos el $10 \%$ de los predios autorizados en cada uno de los seis métodos de planeación principales que se aplican en Durango. Sin embargo, debido a que el MMOBI y el MDS son los más utilizados y en los que mejor se diferencia el sistema de manejo forestal, en este estudio se

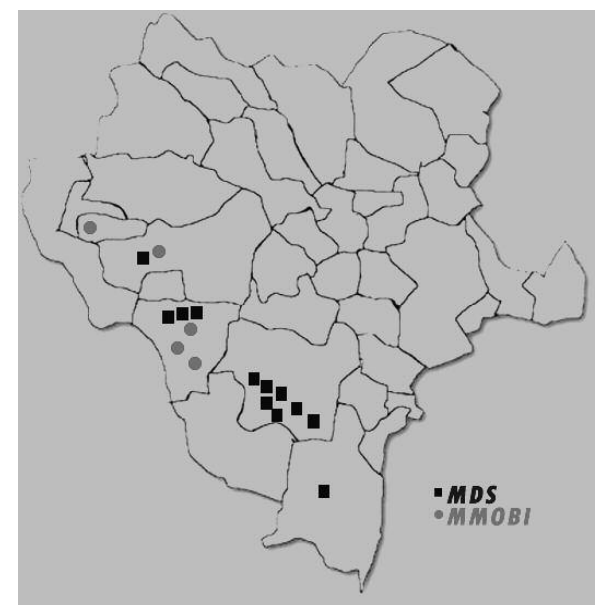

Figura 1. Localización de los predios muestra dentro del área de estudio 
incluyó solamente el análisis realizado para estos dos métodos de planeación.

Para seleccionar los predios muestra se estratificaron 229 predios por el método de planeación. Posteriormente se seleccionaron al azar al menos $10 \%$ de los predios dentro de cada estrato (método de planeación), asegurándose de incluir por lo menos tres predios para cada método, quedando la muestra como se aprecia en la figura 2. La intensidad de muestreo utilizada en el campo fue del $5 \%$ de la superficie para cada unidad básica de manejo (la cual fue el estrato en el caso del MMOBI y el subrodal en el caso del MDS). Un estrato con fines de manejo forestal es considerado como un grupo de rodales que tienen aproximadamente las mismas condiciones de composición y dominancia de géneros, la misma cobertura de copa y la misma altura media (SEMARNAP y AMPF, 1998). Por lo tanto, un estrato cubre un área mucho más extensa y diversa, comparada con el área de un subrodal utilizado como unidad básica de manejo en el MDS.

\section{Datos de campo}

La toma de datos de campo se realizó en el año 2004, seleccionando al azar un número de unidades básicas de manejo, suficientes para completar un máximo de 80 hectáreas por predio, que fue el área factible de estudiar con la intensidad de muestreo programada, dados los recursos financieros disponibles. Dentro de cada unidad básica de manejo se levantaron en forma sistemática sitios circulares de $1000 \mathrm{~m}^{2}$, utilizando una malla de puntos con una separación de $100 \mathrm{~m}$, y levantando un mínimo de tres sitios por unidad. Un total de 46 unidades de manejo distribuidas en 17 predios fueron muestreadas, para evaluar los dos sistemas de manejo forestal (Figura 2). La tabla 1 muestra un resumen de las características de los predios manejados con MDS y $\mathrm{MMOBI}$ en la anualidad 1995-96, considerados en este trabajo.

En cada sitio de muestreo se registraron los siguientes datos: nombre del

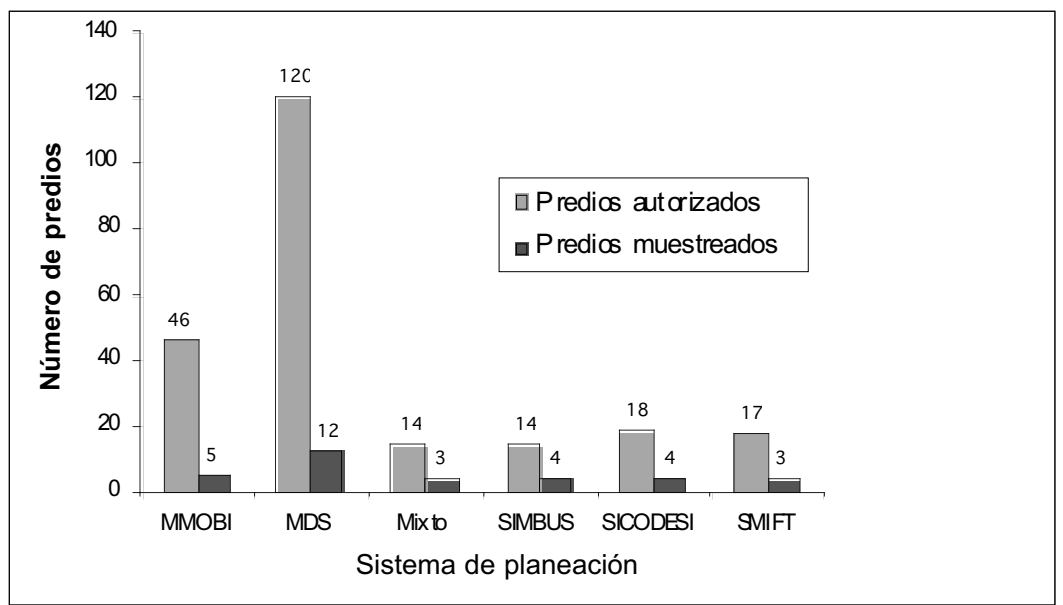

Figura 2. Número de predios autorizados y muestreados para cada sistema de planeación 
Tabla 1. Predios manejados con MMOBI y con MDS en la anualidad 1995-1996 y que se analizaron en este estudio

\begin{tabular}{|c|c|c|c|c|c|c|}
\hline Método y clave por predio & $\begin{array}{c}\text { Superficie } \\
\text { comercial } \\
\text { maderable (ha) }\end{array}$ & $\begin{array}{l}\text { Área de corta } \\
1995-96 \text { (ha) }\end{array}$ & $\begin{array}{c}\text { Área } \\
\text { muestreada } \\
\text { en } 2004 \text { (ha) }\end{array}$ & $\begin{array}{l}\text { Unidades de } \\
\text { manejo } \\
\text { muestreadas } \\
\text { en } 2004\end{array}$ & $\begin{array}{c}\text { Número } \\
\text { de sitios } \\
\text { de } \\
\text { muestreo }\end{array}$ & $\begin{array}{c}\text { Intensidad } \\
\text { de muestreo } \\
\text { en el área } \\
\text { muestreada } \\
(\%)\end{array}$ \\
\hline P.P. El Alamito & 373 & 62 & 62 & 1 & 20 & 3,2 \\
\hline P.P. L-6 Bajío de las Flores & 304 & 92 & 80 & 3 & 40 & 5,0 \\
\hline P.P. Fracc. Segregado P.R. Miravalles & 114 & 71 & 66 & 2 & 35 & 5,3 \\
\hline P.P. Mitad SW L-11 Fracc. P. Miravalles & 100 & 47 & 47 & 4 & 24 & 5,1 \\
\hline Com. San Antonio, Topia & 495 & 101 & 92 & 4 & 40 & 4,3 \\
\hline P.P. Bajios de Don Victor L-7 & 972 & 148 & 83 & 8 & 40 & 4,8 \\
\hline P.P. El Castillito & 278 & 30 & 30 & 2 & 15 & 5,0 \\
\hline P.P. La Ciudad L-1 & 3553 & 586 & 80 & 4 & 39 & 4,9 \\
\hline Ejido El Encinal & 3982 & 233 & 80 & 2 & 40 & 5,0 \\
\hline P.P. Ibáñez L-4 & 472 & 43 & 43 & 3 & 21 & 4,9 \\
\hline P.P. Ibáñez L-5 & 230 & 32 & 32 & 4 & 22 & 6,9 \\
\hline P.P. Laguna Colorada L-52 & 150 & 37 & 37 & 2 & 18 & 4,9 \\
\hline Ejido Navajas & 624 & 58 & 40 & 3 & 21 & 5,3 \\
\hline Ejido Otinapa y San Carlos & 942 & 166 & 133 & 3 & 40 & 3,0 \\
\hline Ejido Regocijo & 6868 & 732 & 80 & 4 & 40 & 5,0 \\
\hline Com. Santa Ma. Magdalena Taxicaringa & 19403 & 1377 & 190 & 4 & 40 & 2,1 \\
\hline NCPE La Victoria, Mpio. Dgo. & 429 & 63 & 35 & 7 & 21 & 6,0 \\
\hline
\end{tabular}

predio, municipio, sistema de manejo, anualidad, responsable técnico, sitio, coordenadas geográficas, número de unidad de manejo, tratamiento silvícola y superficie de la unidad. En los datos dasométricos obtenidos se incluyó información referente al género, especie, diámetro normal y diámetro a la altura del tocón (ambos en categorías de los $5 \mathrm{~cm}$ en adelante), altura total (en categorías de los $5 \mathrm{~m}$ en adelante), edad y tiempo de paso (en años). Como complemento se tomaron datos ecológicos cuantitativos y cualitativos que incluyeron las variables de porcentaje de la pendiente del terreno, la exposición, la altitud y observaciones de perturbaciones evidentes del sitio.

\section{Datos de gabinete}

En los archivos históricos disponibles se revisaron documentos que datan de los años de 1990 para los rodales muestreados en campo, incluyendo: estudios dasonómicos y sus ajustes, planes de manejo integral, programas de manejo forestal, modificaciones e informes finales (SEMARNAT y Prestadores de Servicios Técnicos Forestales, 1990-2004). La información contenida en estos documentos se comparó con la situación reciente reportada en los estudios de la presente década, en los predios que ya contaban con el nuevo programa de manejo. La información se analizó a nivel 
de unidad básica de manejo, incluyendo: la intensidad de muestreo aplicada en el inventario de los años de 1990, el tratamiento silvícola utilizado, las existencias reales totales de pino (en metros cúbicos rollo total árbol por hectárea), la altura media (en metros) y el diámetro normal promedio del arbolado (en centímetros).

La comparación estadística entre la estimación de volumen de pino en pie expresado en metros cúbicos rollo total árbol por hectárea $\left(\mathrm{m}^{3} \mathrm{rta} / \mathrm{ha}\right)$, reportado en los estudios elaborados en los años de 1990, con los datos obtenidos en el muestreo de campo de 2004 se realizó, mediante el uso del intervalo de confianza, calculado para el volumen medio de los datos mediante la ecuación [1]. El intervalo de confianza se define por un límite inferior y un límite superior, dentro de los cuales se espera que se encuentre el valor verdadero del volumen para la población, de acuerdo a un nivel o coeficiente de confianza definido por Merril y Fox (1977). En este trabajo se utilizó un nivel de confianza del 95\%, aceptando un error $\alpha$ del $5 \%$.

$$
I C \equiv \bar{y}_{i} \pm t_{\alpha / 2, v} * S_{y_{i}}
$$

Donde: IC es el valor del intervalo de confianza en $\mathrm{m}^{3} \mathrm{rta} / \mathrm{ha}, \bar{y}$ es la estimación del volumen medio en la unidad básica de manejo $i$ ( $\left.\mathrm{m}^{3} \mathrm{rta} / \mathrm{ha}\right), t$ es el valor del estadístico $t$ de student, $\alpha$ es el error que se acepta tolerar, $v$ son los grados de libertad, y $S_{\bar{y} i}$ es el error estándar del volumen medio en la unidad de manejo $i$ ( $m^{3}$ rta/ha). Los datos se procesaron con el procedimiento MEANS del programa SAS/STAT ${ }^{\circledR}$ (SAS Institute Inc., 2004).

\section{RESULTADOS Y DISCUSIÓN}

\section{Disponibilidad de antecedentes en archivos}

En los programas de manejo revisados, se detectó que en la planeación de las actividades a realizar no se consideraron los resultados obtenidos de la ejecución del programa anterior, ya que no se tomaron en cuenta los informes finales de las anualidades del ciclo de corta anterior. Por otra parte, en la mayoría de los informes finales encontrados de la anualidad de estudio, no se especificaron las acciones realizadas ni los sucesos incontrolables ocurridos en cada unidad básica de manejo, sino solamente a nivel general del área de corta. Por lo tanto, de la revisión de la disponibilidad de antecedentes en archivos se deduce que, hasta el momento, para los predios estudiados no es posible efectuar un seguimiento cuantitativo del historial de manejo para cada uno de los estratos y subrodales, lo cual representó una barrera para lograr el objetivo propuesto.

\section{Cambios en el sistema de planeación, el turno y el ciclo de corta}

En 11 de los 17 predios analizados que en la anualidad 1995-1996 se manejaron con cualquiera de los dos métodos (el MDS o el MMOBI), se encontró que había cambiado el sistema de planeación de la corta para el año 2004 (Tabla 2). Los principales argumentos citados en los documentos para justificar estos cambios fueron: 1) lograr eficazmente los objetivos del manejo, 2) recuperación oportuna de los volúmenes aprovechados, 3) evitar cortas severas en lugares con suelos frágiles y pendientes pronunciadas, 4) disminuir las necesidades de caminos, 5) atender necesidades del mercado y 6) promover la permanencia del empleo local. En siete de los 12 predios manejados 
con MDS en los años de 1990 se encontró información de archivo que permitió comparar la longitud del turno y del ciclo de corta (Tabla 2), advirtiéndose que en cuatro de esos siete predios hubo variaciones en los parámetros silvícolas, así como cambios del sistema de planeación.

Los cambios de sistema de planeación podrían estar justificados por argumentos como los citados. Sin embargo, el aumento o la reducción del ciclo de corta y sobre todo del turno, en periodos de planeación consecutivos, implican el aumento o reducción del volumen de corta sustentable por hectárea en cada intervención, dificultando así su monitoreo a largo plazo (Ludwig et al., 2001). Asimismo, en diversos trabajos se plantea la importancia de mantener constante la delimitación de las unidades básicas de manejo, para poder monitorear con eficiencia los efectos del aprovechamiento sobre la sustentabilidad de variables como la estructura y composición, la biodiversidad y la calidad del ecosistema en general (Putz, 1994; Thoms y Betters, 1998 y Velázquez et al., 2001).

Tabla 2. Predios muestreados incluyendo aquellos para los cuales se detectó un cambio de método de planeación de acuerdo con la información de gabinete

\begin{tabular}{|c|c|c|c|c|c|}
\hline Nombre del predio & Turno (años) & $\begin{array}{c}\text { Ciclo de } \\
\text { corta } \\
\text { (años) }\end{array}$ & $\begin{array}{l}\text { Método de } \\
\text { planeación en } \\
2004\end{array}$ & $\begin{array}{l}\text { Turno } \\
\text { (años) }\end{array}$ & $\begin{array}{c}\text { Ciclo de } \\
\text { corta } \\
\text { (años) }\end{array}$ \\
\hline \multicolumn{6}{|c|}{ Predios manejados con MMOBI en 1995-96 } \\
\hline P.P. EI Alamito & 120 & 20 & SIMBUS & n.d. & n.d. \\
\hline P.P. L-6 Bajío de las Flores & n.d. & 10 & MMOBI & n.d. & 12 \\
\hline P.P. Fracc. Segregada P.R. Miravalles & n.d. & 10 & MMOBI & n.d. & n.d. \\
\hline P.P. Mitad SW L-11 Fracc. P. Miravalles & n.d. & 10 & MMOBI & n.d. & n.d. \\
\hline Com. San Antonio, Topia & n.d. & 20 & SICODESI & 75 & 15 \\
\hline \multicolumn{6}{|c|}{ Predios manejados con MDS en 1995-96 } \\
\hline P.P. Bajíos de Don Victor L-7 & 70 & 14 & MDS & n.d. & n.d. \\
\hline P.P. EI Castillito & 74 & 15 & SIMBUS & 120 & 10 \\
\hline P.P. La Ciudad L-1 & 60 & 12 & MIXTO & 52 & 10 \\
\hline Ejido El Encinal & 50 & 10 & MMOBI & 70 & 14 \\
\hline P.P. Ibáñez L-4 & 60 & 12 & MIXTO & 60 & 12 \\
\hline P.P. Ibáñez L-5 & 60 & 12 & MIXTO & 60 & 12 \\
\hline P.P. Laguna Colorada L-52 & 70 & 14 & MIXTO & n.d. & n.d. \\
\hline Ejido Navajas & 50 & 10 & MMOBI & 60 & 12 \\
\hline Ejido Otinapa y San Carlos & 70 & 14 & MIXTO & n.d. & n.d. \\
\hline Ejido Regocijo & 50 & 10 & MIXTO & n.d. & n.d. \\
\hline Com. Santa Ma. Magdalena Taxicaringa & 70 & 14 & MDS & 70 & 14 \\
\hline NCPE La Victoria, Mpio. Dgo. & 70 & 14 & MDS & n.d. & n.d. \\
\hline
\end{tabular}




\section{Cambios en la rodalización}

Existen variaciones en cuanto a la forma de caracterizar y delimitar los rodales, algunos autores opinan que deben tomarse en cuenta sólo características físicas estables del terreno (Davies y Johnson, 1987 y Hernández, 2004), mientras otros asumen que debe considerarse también la homogeneidad de la vegetación (Society of American Foresters,
1958; Duerr et al., 1979; Leuschner, 1984 y Putz, 1994). En los programas de manejo forestal revisados en el presente trabajo, difieren también los criterios usados por cada responsable técnico para definir los rodales o unidades básicas de manejo, e inclusive estos criterios suelen cambiar al paso del tiempo, originando cambios en la rodalización. Se encontró que, de las 25 unidades de manejo muestreadas en

Tabla 3. Cambios en la rodalización entre los programas de manejo forestal (PMF) para dos ciclos de corta consecutivos

\begin{tabular}{|c|c|c|c|c|c|c|}
\hline \multirow{2}{*}{ Predio } & \multicolumn{2}{|c|}{ PMF con anualidad 1995-96 } & \multicolumn{2}{|c|}{ PMF Posterior } & \multicolumn{2}{|c|}{ Diferencia/U.M. } \\
\hline & U.M. & Área (ha) & U.M. & (ha) & (ha) & $(\%)$ \\
\hline P.P. L-6 Bajío de las Flores & Pqll3 & 23,5 & Pqll3 & 20,0 & -4 & -15 \\
\hline P.P. L-6 Bajío de las Flores & PqIV3 & 56,5 & PqN3 & 58,2 & 2 & 3 \\
\hline Com. San Antonio, Topia & Pqll2 & 28,0 & 26 & 66,0 & 38 & 136 \\
\hline Com. San Antonio, Topia & Pqll I2 & 16,0 & . & . & na & na \\
\hline Com. San Antonio, Topia & PqIII3 & 38,3 & . & . & na & na \\
\hline P.P. EI Castillito & 4001 & 20,0 & (15) 14 & 11,5 & -9 & -43 \\
\hline P.P. EI Castillito & 201 & 10,0 & (15) 18 & 14,8 & 5 & 48 \\
\hline P.P. La Ciudad L-1 & 9010 & 17,0 & . & . & na & na \\
\hline P.P. La Ciudad L-1 & 1205 & 20,0 & 10701 & 28,5 & 9 & 43 \\
\hline P.P. Ibáñez L-4 & 603 & 17,0 & 603 & 27,1 & 10 & 59 \\
\hline P.P. Ibáñez L-4 & 604 & 10,2 & 604 & 23,0 & 13 & 125 \\
\hline P.P. Ibáñez L-4 & 604 & 11,0 & 604 & 5,2 & -6 & -53 \\
\hline P.P. Ibáñez L-5 & 601 & 8,8 & 601 & 8,8 & 0 & 0 \\
\hline P.P. Ibáñez L-5 & 602 & 15,0 & 602 & 18,0 & 3 & 20 \\
\hline P.P. Ibáñez L-5 & 603 & 1,0 & 603 & 6,0 & 5 & 500 \\
\hline P.P. Ibáñez L-5 & 606 & 10,0 & 606 & 10,0 & 0 & 0 \\
\hline Ejido Otinapa y San Carlos & 901 & 74,5 & 901 & 74,5 & 0 & 0 \\
\hline Ejido Otinapa y San Carlos & 1002 & 26,5 & . & . & na & na \\
\hline Ejido Otinapa y San Carlos & 1103 & 32,5 & 1103 & 32,5 & 0 & 0 \\
\hline Ejido Regocijo & 2034 & 12,0 & 16007 & 42,0 & 30 & 250 \\
\hline Ejido Regocijo & 2034 & 33,0 & & . & na & na \\
\hline Ejido Regocijo & 2039 & 4,0 & 16001 & 25,0 & 21 & 525 \\
\hline Ejido Regocijo & 2039 & 42,0 & & . & na & na \\
\hline Ejido Regocijo & 2041 & 23,0 & 16004 & 14,5 & -9 & -37 \\
\hline Ejido Regocijo & 2048 & 22,0 & 14012 & 47,0 & 25 & 114 \\
\hline
\end{tabular}

U.M. = Unidad básica de manejo; na = no aplicable, puesto que en los dos programas de manejo revisados no se encontró la U.M. correspondiente. 
ocho predios, sólo cuatro unidades mantuvieron las mismas dimensiones; en 15 se aumentó o disminuyó la superficie entre un $3 \%$ y un $525 \%$, y en los seis casos restantes ya no aparecieron las unidades anteriores o sus correspondientes en el PMF del ciclo de corta revisado (Tabla 3). Esta situación impidió realizar en todas las unidades muestreadas la evaluación propuesta en este trabajo y también dificulta conocer en el mediano y largo plazo los resultados del manejo forestal.

En la figura 3 se observa un ejemplo del cambio en la rodalización de un predio, entre un programa de manejo forestal de los años de 1990 y uno del año 2001. Estos cambios en la delimitación y extensión de las unidades de manejo de un ciclo de corta al siguiente, limitan drásticamente la posibilidad de dar el seguimiento requerido respecto a la sustentabilidad de las prácticas de manejo forestal, sin importar el sistema de planeación aplicado (Vanclay, 1996 y Gadow et al., 2004). Esto significa que mientras persistan las condiciones encontradas en el presente análisis, es imposible conocer la respuesta del bosque a dichas prácticas.

\section{Comparación del volumen en pie}

Los resultados de la comparación del volumen en pie indican que existen diferencias tanto negativas (i. e. sobrestimaciones) como positivas (i. e. subestimaciones) en la estimación de este
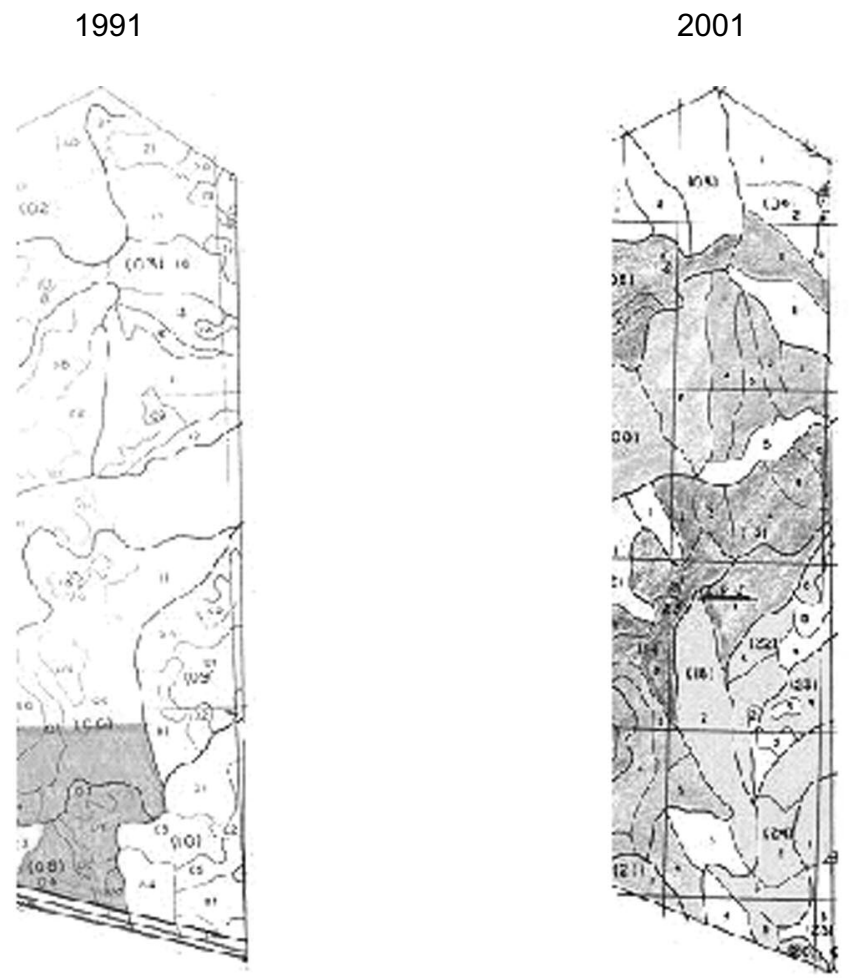

Figura 3. Ejemplo de una sección de un predio mostrando el cambio en la delimitación de los rodales y subrodales en dos ciclos de corta consecutivos 
parámetro dasométrico. Las diferencias varían desde $-51,2 \%$ hasta $331,4 \%$. En 25 de las 46 unidades de manejo analizadas se encontraron diferencias significativas según el intervalo de confianza estimado a partir del muestreo de campo (Tabla 4).

\section{Diferencias en volumen en el MMOBI}

En seis de los 12 estratos evaluados se encontraron diferencias significativas en el cálculo de las existencias reales totales (ERT) de volumen de madera por hectárea (Tabla 4). En algunos estratos, por ejemplo en el llamado Pqll3 del P.P. L6 Bajío de las Flores, se reportaron ERT de $61,9 \mathrm{~m}^{3} \mathrm{rta} / \mathrm{ha}$ en los años de 1990, y según los antecedentes de manejo revisados en esta unidad de manejo se cortaron 10,6 $\mathrm{m}^{3} \mathrm{rta} / \mathrm{ha}$, quedando un volumen residual de 51,3 $\mathrm{m}^{3} \mathrm{rta} / \mathrm{ha}$. La estimación de las ERT en el muestreo del 2004 fue de 110,6 $\mathrm{m}^{3} \mathrm{rta} / \mathrm{ha}$ ( \pm 27,5 $\mathrm{m}^{3} \mathrm{rta} / \mathrm{ha}$ ), lo que implica que el volumen residual de esta unidad de manejo aumentó más del $100 \%$ en tan sólo nueve años. Dado el volumen residual de esta unidad de manejo y sabiendo que el incremento promedio en los bosques comerciales de Durango es del orden de un metro cúbico por hectárea por año (SEMARNAP, 2000), se asume que el incremento de más de $6,5 \mathrm{~m}^{3} \mathrm{rta} / \mathrm{ha} / a n ̃ o$ en esta unidad de manejo es simplemente una sobrestimación.

Las grandes diferencias en el cálculo del volumen en pie se deben entre otras causas a la gran extensión de los estratos, que cubrían entre 15 y más de 50 hectáreas, así como a la baja intensidad de muestreo utilizada en los estudios de los años de 1990. El tamaño de los estratos por un lado pudo haber causado que los sitios muestreados de uno y otro inventario se hayan establecido en comunidades vegetales muy distintas. Ya que aunque la definición de estrato considera condiciones de vegetación homogéneas, en campo se detecta que esta característica difícilmente se cumple y por otra parte las diferencias en el tamaño de las unidades de manejo suponen varianzas muy distintas en el cálculo del volumen.

\section{Análisis de las diferencias en volumen en el MDS}

En 19 de los 34 subrodales analizados en el MDS, se encontraron diferencias significativas entre las ERT reportadas en los estudios de los años de 1990 y las calculadas con los datos del muestreo de 2004 (Tabla 4). Al igual que en el MMOBI, una de las posibles causas de las grandes diferencias encontradas en las ERT/ha es el cambio en las delimitaciones y extensiones de las unidades de manejo, que se observaron en 2004 con relación a los años de 1990 (Tabla 3). Al comparar los planos de programas de manejo sucesivos para un mismo predio, se identificó que algunos rodales y subrodales se subdividieron y otros se modificaron en forma y extensión, afectando la estimación del volumen promedio por hectárea calculados en uno y otro inventario (Figura 3).

En siete de los 12 predios estudiados del MDS se analizó la metodología utilizada para realizar el inventario de manejo y se detectó que en dicho método silvícola se usaron sitios de dimensiones variables para calcular, con apoyo del relascopio, el área basal por hectárea, aplicando un factor de área basal (FAB) previamente determinado y generalizado para todo el predio, mediante el procedimiento estándar conocido en el medio forestal como "hectárea tipo". Los FAB más comunes utilizados para el pino en los estudios revisados fueron el 1 y el 2, y se observó que en ambos casos se asumió que la superficie promedio 
Tabla 4. Comparación de volumen de pino en pie (ERT) entre los años 1990 y 2004

\begin{tabular}{|c|c|c|c|c|c|}
\hline Predio & $\begin{array}{l}\text { Unidad de } \\
\text { manejo }\end{array}$ & $\begin{array}{l}\text { ERT 90's } \\
\left(\mathrm{m}^{3} \mathrm{rta} / \mathrm{ha}\right)\end{array}$ & $\begin{array}{l}\text { ERT } 2004 \\
\text { (m 3rta/ha) }\end{array}$ & Diferencias (\%) & $\begin{array}{l}\text { IC de las } \\
\text { ERT } 2004 \\
\left(\mathrm{~m}^{3} \mathrm{rta} / \mathrm{ha}\right)\end{array}$ \\
\hline P.P. EI Alamito & PqIII3 & 103,5 & 108 & 4,3 & 27,2 \\
\hline P.P. L-6 Bajío de las Flores & Pqll3 & 61,9 & 110,6 & 78,6 & $27,5^{\star}$ \\
\hline P.P. L-6 Bajío de las Flores & PqN3 & 114,4 & 62,4 & $-45,4$ & $8,9^{*}$ \\
\hline P.P. Fracc. Segregada P.R. Miravalles & Pqll2 & 61,5 & 59,3 & $-3,6$ & 11,6 \\
\hline P.P. Fracc. Segregada P.R. Miravalles & PqIII2 & 66,2 & 62,5 & $-5,6$ & 12,9 \\
\hline P.P. Mitad SW L-11 Fracc P. Miravalles & Pqll2 & 61,6 & 69,3 & 12,5 & 30,5 \\
\hline P.P. Mitad SW L-11 Fracc P. Miravalles & PqIII2 & 66,2 & 64,3 & $-2,8$ & 25,2 \\
\hline P.P. Mitad SW L-11 Fracc P. Miravalles & PqIII3 & 79,1 & 46,7 & $-40,9$ & $22,2^{*}$ \\
\hline P.P. Mitad SW L-11 Fracc P. Miravalles & PqIV2 & 93,8 & 46,5 & $-50,4$ & $5,9^{*}$ \\
\hline Com. San Antonio, Topia & Pqll2 & 82,7 & 145,8 & 76,3 & $40,1^{*}$ \\
\hline Com. San Antonio, Topia & PqIII2 & 102,5 & 177 & 72,7 & $60,0^{*}$ \\
\hline Com. San Antonio, Topia & PqIII3 & 172,2 & 193,7 & 12,5 & 58,2 \\
\hline Promedio & & 88,8 & 95,5 & 9 & 27,5 \\
\hline PP Bajíos de Don Victor L-7 & 1010 & 60,7 & 118,6 & 95,4 & $35,0^{*}$ \\
\hline PP Bajíos de Don Victor L-7 & 1012 & 73,2 & 95,5 & 30,5 & 58 \\
\hline PP Bajíos de Don Victor L-7 & 1015 & 55,8 & 60,7 & 8,8 & 14,3 \\
\hline PP Bajíos de Don Victor L-7 & 1019 & 49,7 & 72,6 & 46,1 & 28,5 \\
\hline PP Bajíos de Don Victor L-7 & 1101 & 66,2 & 43,9 & $-33,7$ & $22,1^{*}$ \\
\hline PP Bajíos de Don Victor L-7 & 1108 & 69,3 & 52,2 & $-24,7$ & 22,1 \\
\hline PP Bajíos de Don Victor L-7 & 1205 & 110,6 & 180 & 62,8 & $6,3^{*}$ \\
\hline PP El Cas tillito & 201 & 46,8 & 94,8 & 102,5 & $30,0^{*}$ \\
\hline PP El Cas tillito & 4001 & 78,9 & 100,5 & 27,4 & 27,2 \\
\hline PP La Ciudad L-1 & 9010 & 181,1 & 149,3 & $-17,6$ & $39,1^{*}$ \\
\hline PP La Ciudad L-1 & 1205 & 108,5 & 113,4 & 4,5 & 45,1 \\
\hline Ejido El Encinal & 1104 & 51,7 & 84,6 & 63,6 & $11,1^{*}$ \\
\hline Ejido El Encinal & 1105 & 69 & 110,4 & 60 & $37,9^{*}$ \\
\hline PP Ibáñez L-4 & 603 & 163,3 & 230,3 & 41 & $48,4^{*}$ \\
\hline PP lbáñez L-4 & 604 & 194,8 & 144,3 & $-25,9$ & $47,6^{*}$ \\
\hline PP lbáñez L-5 & 601 & 80 & 73,5 & $-8,2$ & 23 \\
\hline PP lbáñez L-5 & 602 & 80,8 & 105,1 & 30,1 & 29,4 \\
\hline PP Ibáñez L-5 & 603 & 119 & 228,9 & 92,4 & $74,0^{*}$ \\
\hline PP lbáñez L-5 & 606 & 106 & 180,9 & 70,6 & $14,3^{*}$ \\
\hline Ejido Navajas & 1505 & 29,8 & 36,6 & 22,8 & $6,3^{*}$ \\
\hline Ejido Navajas & 1506 & 32,3 & 22,9 & $-29,1$ & $5,8^{*}$ \\
\hline
\end{tabular}


Tabla 4. Continúa

\begin{tabular}{|c|c|c|c|c|c|}
\hline Predio & $\begin{array}{l}\text { Unidad de } \\
\text { manejo }\end{array}$ & $\begin{array}{l}\text { ERT } \frac{90 ' s}{\left(\mathrm{~m}^{3} \mathrm{rta} / \mathrm{ha}\right)} \\
\text {. }\end{array}$ & $\begin{array}{l}\text { ERT } 2004 \\
\text { (m3rta/ha) }\end{array}$ & Diferencias $(\%)$ & $\begin{array}{l}\text { IC de las } \\
\text { ERT 2004 } \\
\left(\mathrm{m}^{3} \text { rta/ha }\right)\end{array}$ \\
\hline Ej. Otinapa y San Carlos & 901 & 62,5 & 36,5 & $-41,7$ & $5,0^{*}$ \\
\hline Ej. Otinapa y San Carlos & 1002 & 53,9 & 29 & $-46,3$ & 15,4 \\
\hline Ej. Otinapa y San Carlos & 1103 & 66 & 56,6 & $-14,3$ & $6,3^{*}$ \\
\hline Ejido Regocijo & 2041 & 70,3 & 85,7 & 21,9 & 26,1 \\
\hline Ejido Regocijo & 2048 & 77,2 & 75,5 & $-2,2$ & 13,6 \\
\hline Com. Santa Ma. Magdalena Taxicaringa & 8401 & 58,9 & 78,4 & 33,1 & 37,4 \\
\hline Com. Santa Ma. Magdalena Taxicaringa & 8403 & 42 & 92,8 & 121 & $35,6^{*}$ \\
\hline Com. Santa Ma. Magdalena Taxicaringa & 8404 & 52 & 105,5 & 103 & $21,7^{*}$ \\
\hline Com. Santa Ma. Magdalena Taxicaringa & 8405 & 87 & 81,9 & $-5,9$ & 33,6 \\
\hline NCPE La Victoria, Mpio. Dgo. & 302 & 50,7 & 80,9 & 59,6 & $24,8^{*}$ \\
\hline NCPE La Victoria, Mpio. Dgo. & 304 & 27,3 & 117,8 & 331,4 & 99,4 \\
\hline NCPE La Victoria, Mpio. Dgo. & 305 & 84,8 & 41,4 & $-51,2$ & $20,5^{*}$ \\
\hline NCPE La Victoria, Mpio. Dgo. & 3010 & 68,7 & 68,3 & $-0,5$ & 47,7 \\
\hline Promedio & & 59,5 & 69,5 & 34,2 & 28,1 \\
\hline
\end{tabular}

* Indica una diferencia significativa entre las ERT/ha de los años 1990's y las encontradas en el año 2004 con · = $5 \% . I C=$ Intervalo de confianza al $95 \%$ para el promedio de las existencias reales totales de pino calculadas en 2004.

cubierta por cada sitio de muestreo de dimensiones variables era de $1000 \mathrm{~m}^{2}$, calculando así el número de sitios necesarios para lograr la intensidad de muestra que se haya prescrito en el área a estudiar.

Para corroborar la validez de los FAB utilizados en los PMF de los años de 1990 , se verificó el diámetro promedio reportado para el género Pinus en siete predios y se determinó el FAB apropiado para lograr que cada sitio de dimensiones variables realmente cubriera un área aproximada de $1000 \mathrm{~m}^{2}$, de acuerdo con la tabla del radio máximo del sitio de dimensiones variables y representación del número de árboles para diferentes factores de área basal reportada por Cano (1988). Se encontró que, según el diámetro promedio del arbolado en los subrodales muestreados, los FAB utilizados en el inventario de los años de
1990, en todos los casos, fueron del doble de magnitud que los FAB apropiados para que cada sitio muestral cubriera $1000 \mathrm{~m}^{2}$ (Tabla 5).

El problema de usar un FAB mayor que el adecuado, es que el radio medio de cada sitio fue menor que $17,84 \mathrm{~m}$, por lo que el área del sitio fue menor que los $1000 \mathrm{~m}^{2}$ asumidos. Por lo tanto, obteniendo la proporción entre el área supuesta y el área real cubierta por cada sitio, se calculó que la intensidad de muestreo que realmente se aplicó en los siete predios analizados, fue menor que la intensidad de muestra que se declaró en los programas revisados. Esta baja intensidad de muestreo, es sin duda, una causa de las grandes diferencias entre los volúmenes de madera de pino en pie reportados para la anualidad 1995-1996 y los volúmenes estimados en el muestreo de campo de 2004. 
Tabla 5. Radio máximo de los sitios de dimensiones variables y representación del número de árboles para diferentes factores de área basal (FAB)

\begin{tabular}{|c|c|c|c|c|c|c|c|c|c|}
\hline \multirow{2}{*}{$\begin{array}{c}\text { Diámetro } \\
\text { medio }(\mathrm{cm})\end{array}$} & \multicolumn{3}{|c|}{$\begin{array}{c}\text { Radio en metros, de cada sitio } \\
\text { con cada factor de área basal } \\
\text { (FAB) }\end{array}$} & \multicolumn{3}{c|}{$\begin{array}{c}\text { Nú mero de árboles/ha que } \\
\text { representa cada árbol en el } \\
\text { sitio, según elFAB }\end{array}$} & \multirow{2}{*}{ AB/árbol } \\
\cline { 2 - 8 } & 0,5 & 1 & 2 & 3 & 0,5 & 1 & 2 & 3 & \\
\hline 5 & 3,5 & 2,5 & 1,75 & 1,45 & 260 & 510 & 1020 & 1530 & 0,00196 \\
10 & 7,0 & 5,0 & 3,50 & 2,90 & 64 & 128 & 256 & 584 & 0,00785 \\
15 & 10,6 & 7,5 & 5,25 & 4,35 & 29 & 57 & 114 & 170 & 0,01767 \\
20 & 14,4 & 10,0 & 7,00 & 5,80 & 16 & 32 & 64 & 96 & 0,03144 \\
25 & 17,7 & 12,5 & 8,75 & 7,25 & 10 & 20 & 40 & 60 & 0,04908 \\
30 & 21,2 & 15,0 & 10,50 & 8,70 & 7 & 14 & 28 & 42 & 0,07067 \\
35 & 24,7 & 17,5 & 12,25 & 10,15 & 5 & 10 & 20 & 30 & 0,09622 \\
40 & 28,2 & 20,0 & 14,00 & 11,60 & 4 & 8 & 16 & 24 & 0,12560 \\
45 & 31,8 & 22,5 & 15,75 & 13,05 & 3 & 6 & 12 & 18 & 0,15904 \\
50 & 35,4 & 25,0 & 17,50 & 14,50 & 2,5 & 5 & 10 & 15 & 0,19635 \\
55 & 38,8 & 27,5 & 19,25 & 15,95 & 2 & 4 & 8 & 12 & 0,23758 \\
60 & 42,4 & 30,0 & 21,00 & 17,40 & 1,5 & 3 & 6 & 10 & 0,28260 \\
\hline
\end{tabular}

Fuente: Cano (1988).

Otra explicación de las diferencias en las ERT/ha, aplicable tanto a los predios manejados con el MMOBI como a los manejados con el MDS, es la posibilidad de que se cometan errores sistemáticos en la estimación de los datos de alturas y diámetros, pues las brigadas que toman los datos de campo durante los inventarios para manejo forestal, usualmente estiman los diámetros del arbolado en categorías de $5 \mathrm{~cm}$ en adelante y las categorías de alturas en clases superiores a los $5 \mathrm{~m}$. Según Cano (1988), si a un árbol se le subestima o sobrestima su categoría de diámetro, el volumen de ese árbol puede estarse subestimando o sobrestimando hasta en un $44 \%$ y un $75 \%$, respectivamente. En cuanto al efecto de subestimar o sobrestimar la altura del arbolado en una categoría, cuando estas varían de 5 en 5 metros, los errores en la estimación del volumen alcanzan desde una subestimación del $25 \%$ hasta una sobrestimación del $33 \%$, dependiendo de las dimensiones y del coeficiente mórfico de los árboles de que se trate. Otros autores que han estudiado la influencia de la exactitud de los datos en la estimación del crecimiento del área basal y/o volumen, son McRoberts et al. (1994), Huang y Titus (1995) y Monserud y Sterba (1996). Estos autores señalan que el efecto de la estimación del diámetro puede ser muy importante, especialmente para especies de crecimiento lento, y coinciden en que errores entre el $0,3 \%$ y $1,25 \%$ en la estimación de diámetro real, pueden arrojar estimaciones del crecimiento en área basal con errores entre $4 \%$ y $18 \%$.

\section{CONCLUSIONES}

De la revisión de los antecedentes a los que se tuvo acceso en archivos se concluye que, hasta el momento, para los predios estudiados no es posible efectuar un seguimiento cuantitativo del historial de las unidades básicas de manejo forestal, considerando que en los programas revisados no se mencionan los antecedentes de las prácticas de manejo efectuadas a este nivel, sino que solamente se citan las áreas de corta 
que fueron programadas en el estudio anterior. En 11 de los 17 predios analizados se observaron cambios de sistema de planeación entre el ciclo de corta evaluado y el posterior. Estos cambios generalmente fueron acompañados de modificaciones en la forma y tamaño de la unidades de manejo. Los cambios en estas variables dificultaron la posibilidad de evaluar la sustentabilidad del manejo forestal, ya que con estas acciones no se permitió llevar a cabo con eficiencia el monitoreo, en el mediano y largo plazo, de los efectos del manejo sobre variables dasométricas, económicas y ecológicas.

Al comparar los volúmenes por hectárea estimados con el muestreo de campo del año 2004, con los volúmenes reportados en los programas de manejo elaborados en los años de 1990, en seis de los 12 estratos manejados con el MMOBI y en 19 de los 34 subrodales tratados con MDS, se encontraron diferencias significativas en su estimación. Las diferencias en volumen encontradas en el MMOBI se atribuyen entre otras causas a la extensión de los estratos, que cubrían entre 15 y más de 50 hectáreas, y a la baja intensidad de muestreo utilizada en los estudios de los años de 1990. En ambos métodos las diferencias en volumen se atribuyen además al cambio en la delimitación y extensión de las unidades de manejo, efectuado entre ciclos de corta. Aunque las brigadas responsables de la toma de datos de inventario también afectan la posible subestimación o sobrestimación sistemática de las categorías de diámetro y clases de altura del arbolado. Otra causa adicional, en el caso del MDS, es el uso de factores de área basal no apropiados, con los cuales se obtuvo una intensidad de muestreo que fue en realidad menor que la registrada en los programas de manejo revisados.

Para ayudar a resolver la problemática detectada y así contribuir al manejo forestal sustentable, es necesario conservar a largo plazo la misma delimitación de las unidades básicas de manejo, en particular los rodales, y mejorar los registros técnicos para lograr un seguimiento adecuado de estas unidades. En los informes finales de cada anualidad deben describirse las actividades y sucesos ocurridos en cada unidad básica, y al formular el siguiente programa de manejo forestal de cada predio, deben tomarse en cuenta estos informes e incluirlos como anexos. Para obtener resultados confiables al nivel de cada unidad de manejo, es recomendable utilizar en los inventarios sólo sitios de dimensiones fijas y decidir la intensidad de muestreo con base en la variabilidad de las características del arbolado por inventariar. Asimismo, es importante capacitar y supervisar al personal de campo, para reducir los errores de sub o sobrestimación.

\section{RECONOCIMIENTOS}

Al fondo de investigación CONAFOR-CONACYT por su apoyo al proyecto número 4666. A los responsables de servicios técnicos de los predios muestreados y la SEMARNAT de Durango, quienes aportaron información documental para realizar el trabajo.

\section{REFERENCIAS}

Cano C., J. 1988. El sistema de manejo regular en los bosques de México. División de Ciencias Forestales, $\mathrm{UACH}$. Chapingo, México, $221 \mathrm{p}$.

Corral R., J., O. Aguirre C., J. Jiménez P. y S. Corral R. 2005. Un análisis del efecto del aprovechamiento forestal sobre la diversidad estructural en el bosque mesófilo de montaña "El Cielo", Tamaulipas, México. Investigación Agraria. Sistemas y Recursos Forestales 14(2):217-228. 
Davies, L. S. y K. N. Johnson. 1987. Forest management. Third Edition. McGraw-Hill. 790 p.

Duerr, W. A., D. E. Teeguarden, N. B. Chritiansen y S. Guttenberg (Ed.). 1979. Forest resource management. Decision-making principles and cases. W. B. Saunders. 612 p.

Gadow, K. V., S. Sánchez y O. Aguirre C. 2004. Manejo forestal con bases científicas. Madera y Bosques 10(2):3-16.

González E., M. S., M. González E. y M. A. Márquez L. 2007. Vegetación y eco-regiones de Durango. Plaza y Valdés, México, 219 p.

Hernández D., J. C. 2004. Importancia de los rodales en el manejo forestal sustentable. Universale Forestum. Órgano informativo del ISIMA-UJED, año 3 , núm. 1.

Huang, S. y S. J. Titus. 1995. An individual tree diameter increment model for white spruce in Alberta. Canadian Journal of Forest Research 25:14551465.

Leuschner, W. A. 1984. Introduction to forest resource management. John Wiley. 298 p.

Ludwig, K., P. Köhler y A. Huth. 2001. Sustainable timber harvesting in Venezuela: a modelling approach. Journal of Applied Ecology 38:756770.

Mc Roberts, R. E., J. T. Hahn, G. J. Heftt y J. R. van Cleve. 1994. Variation in forest inventory field measurements. Canadian Journal of Forest Research 24:1766-1770.

Mendoza M., R. y R. Rodríguez C. 1959. Crítica de los conceptos edad, turno y diámetro mínimo de corta. Apéndice siete del Proyecto General de Ordenación Forestal de la U.I.E.F. Michoacana de Occidente, S. de R. L. 82 p.

Merril, W. y A. Fox. 1977. Introducción a la estadística económica. Amorrortu. Traducida del inglés por M. Kitaigorodzki, Argentina.

Monserud, R. A. y H. Sterba. 1996. A basal area increment model for individual trees growing in even-aged forest stands in Austria. Forest Ecology and Management 80:57-80.

Pélissier, R., J. P. Pascal, F. Houllier y H. Laborde. 1998. Impact of selective logging on the dynamics of a low elevation dense moist evergreen forest in the Western Ghats (south India). Forest Ecology and Management 105:107-119.

Putz, F. E. 1994. Approaches to sustainable forest management. CIFOR, Working Paper núm. 4.7 p.

SARH. 1994. Inventario forestal periódico del estado de Durango. P. 35-36.

SAS Institute Inc. 2004. SAS/STAT, 9.1.2. User's Guide. Cary, NC: SAS Institute, Inc.

SEMARNAP y AMPF, A.C. 1998. Bases científicas para la elaboración de programas de manejo forestal en bosques de coníferas con fines de producción. Notas del Curso-Taller realizado en Metepec, Pue., México, 30 nov. -14 dic.

SEMARNAP-Durango. 2000. Diagnóstico de la actividad forestal de Durango y sus perspectivas. Documento interno de trabajo de la Subdelegación de Recursos Naturales de la SEMARNAP en Durango, 42 p, inédito. 
Society of American Foresters. 1958. Forest terminology. 3a. ed., Washington, D. C.

Solís M., R., O. Aguirre C., E. J. Treviño G., J. Jiménez P., E. Jurado I. y J. Corral R. 2006. Efecto de dos tratamientos silvícolas en la estructura de ecosistemas forestales en Durango, México. Madera y Bosques 12(2):4964.

Thoms, Ch. A. y D. R. Betters. 1998. The potential for ecosystem management in Mexico's forest ejidos. Forest Ecology and Management 103:149-157.

Torres R., J. M. 1999. Sostenibilidad del volumen de cosecha calculado con el Método de Ordenación de Montes. Doc. de trabajo núm. 149. CIDE. 23 p.
Vanclay, J. K. 1996. Estimating sustainable timber production from tropical forests. CIFOR Working Paper No. 11. Estimating AAC in the Tropics.

Velázquez, A., G. Bocco y A. Torres. 2001. Turning scientific approaches into practical conservation actions: The case of Comunidad indígena de Nuevo San Juan Parangaricutiro, México. Environmental Management 27(5):655-665. 
